\title{
Robotic esophagectomy: how I do it?
}

\author{
Puja Gaur Khaitan ${ }^{1,2}$, John F. Lazar ${ }^{1,2}$, Marc Margolis ${ }^{1,2}$, Hayley R. Henderson ${ }^{2}$, Thomas J. Watson ${ }^{1,2}$ \\ 'Department of Surgery, Division of Thoracic and Esophageal Surgery, Georgetown University School of Medicine, MedStar \\ Washington Hospital Center, Washington, DC 20010, USA. \\ 2Division of Thoracic and Esophageal Surgery, Georgetown University School of Medicine, MedStar Washington Hospital \\ Center, Washington, DC 20010, USA.
}

Correspondence to: Dr. Puja Gaur Khaitan, Department of Surgery, Division of Thoracic and Esophageal Surgery, Georgetown University School of Medicine, MedStar Washington Hospital Center, 110 Irving Street, NW (G253), Washington, DC 20010, USA. E-mail: puja.g.khaitan@medstar.net How to cite this article: Khaitan PG, Lazar JF, Margolis M, Henderson HR, Watson TJ. Robotic esophagectomy: how I do it? Mini-
invasive Surg 2020;4:51. http://dx.doi.org/10.20517/2574-1225.2020.34

Received: 9 Apr 2020 First Decision: 19 May 2020 Revised: 6 Jun 2020 Accepted: 17 Jun 2020 Published: 15 Aug 2020

Academic Editor: Noriyoshi Sawabata Copy Editor: Cai-Hong Wang Production Editor: Jing Yu

\begin{abstract}
Compared to the open approach, minimally invasive esophagectomy (MIE) offers several advantages including smaller incisions with decreased pain, improved cosmesis, and earlier return of the patient to baseline function. Robotic-assisted minimally invasive esophagectomy (RAMIE) builds on standard MIE by offering threedimensional visualization, better instrument articulation, tremor filtration, and superior ergonomics, all of which facilitate technical precision and surgeon comfort. An evolving literature demonstrates that when performed by experienced surgeons, RAMIE leads to improved perioperative outcomes with long-term oncologic equivalency to open approaches, and may offer advantages compared to traditional MIE. This review focuses on the key steps of performing 3-field McKeown, 2-field Ivor Lewis, and transhiatal robotic esophagectomies, data regarding the short- and long-term outcomes, and a brief overview of upcoming trials comparing RAMIE with MIE.
\end{abstract}

Keywords: Esophagectomy, robotic, minimally invasive esophagectomy

\section{INTRODUCTION}

Over the last two decades or more, minimally invasive approaches to esophagectomy have been adopted with increasing frequency. The benefits of minimally invasive surgery include smaller incisions, less pain, improved cosmesis, decreased lengths of stay, and quicker return of the patient to baseline function. When performed for thoracic malignancies, minimally invasive resections have led to cure rates equivalent to

cC) (C) The Author(s) 2020. Open Access This article is licensed under a Creative Commons Attribution 4.0 International License (https://creativecommons.org/licenses/by/4.0/), which permits unrestricted use, sharing, adaptation, distribution and reproduction in any medium or format, for any purpose, even commercially, as long as you give appropriate credit to the original author(s) and the source, provide a link to the Creative Commons license, and indicate if changes were made. 
those found from open procedures ${ }^{[1-5]}$. These results have translated more recently to robotic surgery as well. The first minimally invasive esophagectomy (MIE) was reported in $1992^{[6]}$, and the first roboticassisted minimally invasive esophagectomy (RAMIE) with an intrathoracic anastomosis was published in $2002^{[7]}$. This report was followed by the first transhiatal robotic esophagectomy in 2003 and the first McKeown robotic esophagectomy with cervical anastomosis in $2004^{[8,9]}$. Since then, numerous authors have reported the perioperative safety, efficacy, and potential advantages of robotic-assisted esophageal resection.

The early experience with RAMIE suggested an increased incidence of complications, including anastomotic leaks and conduit loss ${ }^{[10-13]}$, compared to open and traditional MIE approaches. The more recent literature, however, has shown a complication profile comparable to $\mathrm{MIE}^{[2,14,15]}$. This evolution in outcomes following RAMIE is likely due to the steep learning curve associated with the introduction of a new technology, especially for a complex operation such as esophagectomy. Improvements over time may also be attributable to the adoption of structured protocols for the teaching and proctoring of robotic operations intended to enhance surgeon proficiency and safety ${ }^{[16]}$.

This review will focus on the technical details of performing and outcomes following 2-field, 3-field, and transhiatal RAMIE, including recent and ongoing studies, as well as potential future trends.

\section{INDICATIONS FOR SURGERY}

The indications for RAMIE are the same as for open esophagectomy or traditional MIE, including esophageal cancer, Barrett's esophagus with high-grade dysplasia (unamenable to, or having failed, endoscopic therapy), recalcitrant esophageal stricture, and end-stage achalasia. A contraindication to RAMIE is the presence of extensive thoracic or abdominal adhesions that preclude a minimally invasive approach. In addition, if the stomach has been resected or its vascularity interrupted by prior surgery, an alternate esophageal replacement conduit, such as the colon or jejunum, may be required. An open operation may be necessary in such cases, as the experience with robotic approaches to utilizing conduits other than the stomach is limited.

\section{TWO-FIELD RAMIE (IVOR LEWIS RAMIE)}

\section{Patient positioning and abdominal port placement}

An Ivor Lewis RAMIE is started with the patient supine. Four robotic ports (one $12 \mathrm{~mm}$ and three $8 \mathrm{~mm}$ ) are typically employed as seen in Figure 1A. A $12 \mathrm{~mm}$ right upper quadrant port is needed to create a gastric conduit with the use of a robotic stapler. The abdomen is entered with either a Hassan or Optiview technique using a 0 -degree, $5 \mathrm{~mm}$ camera in the left upper quadrant. This port is later converted to an $8 \mathrm{~mm}$ robotic port. Once the peritoneal cavity is entered, carbon dioxide is insufflated to a sustained pressure of $15 \mathrm{mmHg}$. The remaining ports are placed under direct visualization. Three other robotic ports (one $8 \mathrm{~mm}$ midline, one $8 \mathrm{~mm}$ left lateral quadrant, and one $12 \mathrm{~mm}$ right upper quadrant) are employed. These incisions are all equidistant from the xiphoid process. A $5 \mathrm{~mm}$ liver retractor port can be placed either laterally in the right upper quadrant or near the subxiphoid process. Finally, an assistant port is positioned low in the pelvis, typically on the patient's right, to facilitate placement of a feeding jejunostomy in the left lower quadrant. We use a $12 \mathrm{~mm}$ valveless insufflation port (AirSeal; Conmed, Utica, NY) as our assistant port. It serves the dual roles of providing controlled air insufflation while being sufficiently large to allow passage of cigar-shaped sponges, topical hemostatic agents, and Penrose drains throughout the case.

\section{Abdominal portion of the procedure}

Following thorough exploration for metastatic disease, abdominal dissection begins by creating the gastric conduit. The right gastroepiploic artery is identified within the greater omentum [Figure 1B]. Starting at the level of the pylorus, the gastrocolic ligament is divided along the greater curvature of the stomach using 

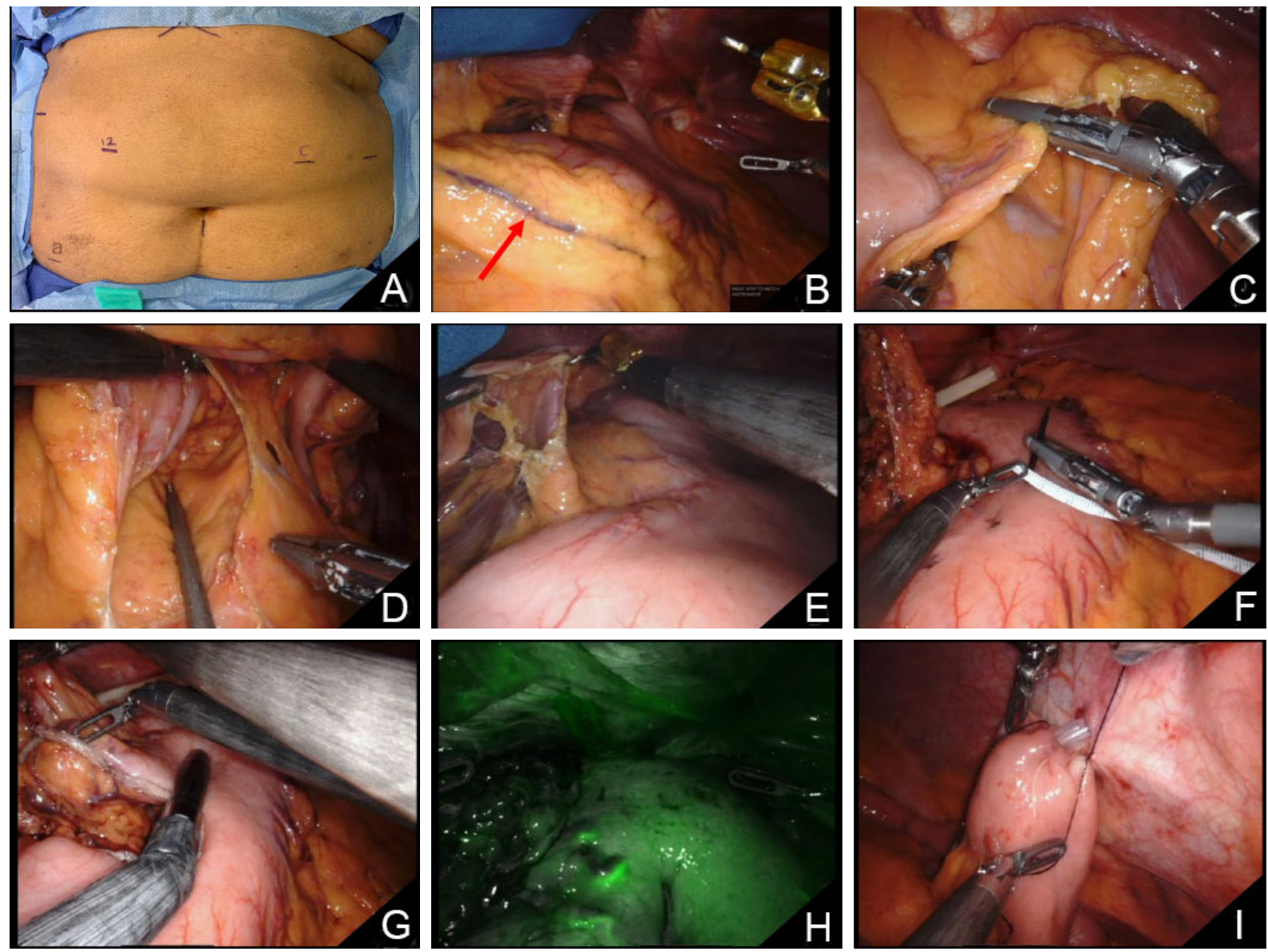

Figure 1. Abdominal portion of robotic Ivor Lewis esophagectomy. A depiction of abdominal robotic port placement is demonstrated [A: (c) camera port; (a) assistant port]; first, the right gastroepiploic arcade is defined ( $B$, red arrow); the greater omentum is then divided and dissection proceeds along the short gastric vessels (C); once the left crus is defined, the stomach is rotated and retrogastric attachments are divided (D); the gastrohepatic ligament is divided (E) and the right crus is defined; a 4-5 cm gastric conduit is created (F) with serial firing of staplers $(\mathrm{G})$; indocyanine green can be used to define the vascularity of the conduit $(\mathrm{H})$; finally, a jejunostomy feeding tube is placed in the left lower quadrant (I) prior to abdominal closure

a robotic vessel sealer, taking care to preserve the right gastroepiploic arcade. Once the right gastroepiploic trunk tapers proximally along the greater curvature, the dissection plane is moved closer to the stomach and the short gastric vessels are ligated [Figure 1C]. After division of the gastrocolic ligament in its entirety, posterior dissection of the stomach is completed [Figure 1D]. The left and right crura are identified and the overlying peritoneum is dissected and swept towards the specimen. The pars flaccida (gastrohepatic ligament) is divided, taking care to preserve any accessory branches of the left hepatic artery, if sizeable [Figure 1E]. The left gastric artery is identified, nodes at its base are swept up with the specimen, and the vessel is transected flush against the celiac axis using a robotic curved tip vascular white load stapler. As mediastinal dissection is performed, a Penrose drain may be placed around the lower esophagus for later retrieval from the chest.

Next, a gastric conduit is created by dividing the lesser curvature of the stomach. A ruler may be used by the surgeon to measure a $4-5 \mathrm{~cm}$ transverse diameter conduit [Figure $1 \mathrm{~F}$ ], and then created with serial firings of blue- or green-load $45 \mathrm{~mm}$ robotic staplers. The gastric conduit is not transected in its entirety from the proximal stomach until further dissection is performed in the chest [Figure $1 \mathrm{G}$ ]. Maintaining a connection between the specimen and the conduit allows the latter to be delivered into the mediastinum in the proper orientation as the esophagus is brought out through a subsequent chest incision. Using 
the near-infrared imaging mode on the robotic console, indocyanine green (ICG) may be administered intravenously (IV) to define the vascularity of the conduit [Figure $1 \mathrm{H}$ ]. The literature is equivocal on the use of ICG, as there is no conclusive evidence demonstrating decreased anastomotic leak rates with this strategy ${ }^{[17,18]}$. We typically use $5 \mathrm{mg}$ of ICG injected IV by the anesthesia team followed by a saline flush. The vascular arcade is analyzed using the near-infrared imaging within one minute after injection. A stitch is placed at the transition point where a loss of perfusion is noted in the gastric conduit. A HeinekeMikulicz pyloroplasty is performed routinely by opening the anterior aspect of the pylorus longitudinally and closing it transversely using running 2-0 Ethibond stitches (Ethicon Inc., Somerville, NJ). The suture line is buttressed with a tongue of omentum. A 12-14 Fr feeding jejunostomy tube is placed in the proximal jejunum and brought out to the skin through a small incision in the left anterior abdominal wall [Figure 1I].

\section{Thoracic portion of the procedure and anastomotic development}

After completion of the abdominal phase, the patient is reintubated with a double lumen endotracheal tube and placed in the left lateral decubitus position. Single lung ventilation is established in the left lung. Four robotic ports are used to facilitate thoracic mobilization of the esophagus in addition to a fifth valveless insufflation assistant port [Figure 2A]. Three robotic ports are placed in the eighth intercostal space starting anterior to the anterior axillary line $(12 \mathrm{~mm})$, posterior axillary line $(8 \mathrm{~mm})$, and posteriorly approximately $2 \mathrm{~cm}$ away from the spine $(8 \mathrm{~mm})$. An $8 \mathrm{~mm}$ robotic port is placed in the third or fourth intercostal space anteriorly to allow for upper esophageal mobilization. An assistant $12 \mathrm{~mm}$ valveless insufflation port is placed low in the pleural cavity at about the tenth intercostal space at the level of the diaphragm. The chest is typically insufflated with carbon dioxide to a pressure of 8-10 $\mathrm{mmHg}$.

Using a curved bipolar instrument, circumferential esophageal mobilization is performed starting from the level of the hiatus, proceeding superiorly to the level of the azygos vein. Starting at the inferior pulmonary ligament [Figure $2 \mathrm{~B}$ ], all paraesophageal lymphoid tissue is either removed serially during the dissection or included with the surgical specimen. Any subcarinal nodal tissue is also dissected; the bronchus intermedius is typically skeletonized in the process [Figure $2 \mathrm{C}$ ]. The abdominal Penrose drain is retrieved and pulled into the chest, ensuring circumferential dissection of the esophagus [Figure 2D]. After mobilization of the esophagus towards the thoracic inlet, the azygos vein is divided near the superior vena cava (SVC) using a white vascular load curved tip robotic stapler [Figure 2E].

Once esophageal dissection is completed, the esophagus is divided approximately $2-3 \mathrm{~cm}$ superior to the azygos vein [Figure $2 \mathrm{~F}$ ] and the gastric conduit is pulled into the chest [Figure $2 \mathrm{G}$ ]. The proximal esophageal margin is sent for frozen section analysis to ensure that it is negative for metaplasia, dysplasia, or malignancy [Figure $2 \mathrm{H}$ ]. A $28 \mathrm{~mm}$ end-to-end anastomotic (EEA) stapler anvil is inserted into the proximal esophagus after removing any staples placed during transection. Running 3-0 vicryl (Ethicon, Somerville, NJ) "baseball stitch" sutures are positioned around the esophageal edge to secure the anvil in the proximal esophagus. An additional reinforcing purse string suture may be employed to assure mucosal apposition around the stem of the anvil during deployment of the stapler. Alternatively, a transoral anvil $\left(\mathrm{OrVil}^{\mathrm{TM}}\right.$, Medtronic, Mansfield, Massachusetts) may be passed through the proximal esophageal staple line [Figure 2I]. The posterior axillary line port is extended into a $4-5 \mathrm{~cm}$ access incision to facilitate completion of the anastomosis (either intracorporeal or extracorporeal; in our case, extracorporeal anastomosis was performed). Using a soft tissue Alexis retractor, this incision can be opened further. A gastrotomy is then created in the proximal conduit tip, and the EEA stapler is introduced through it. Once in appropriate position to engage with the anvil without excessive redundancy in the conduit, the stapler spike is brought out of the greater curvature of the conduit at or below the transition stitch. After appropriate alignment, the stapler is docked onto the anvil and fired, creating the anastomosis. Two mucosal "rings", one esophageal and one gastric, are confirmed in the EEA stapler once it is removed from the thoracic cavity. 

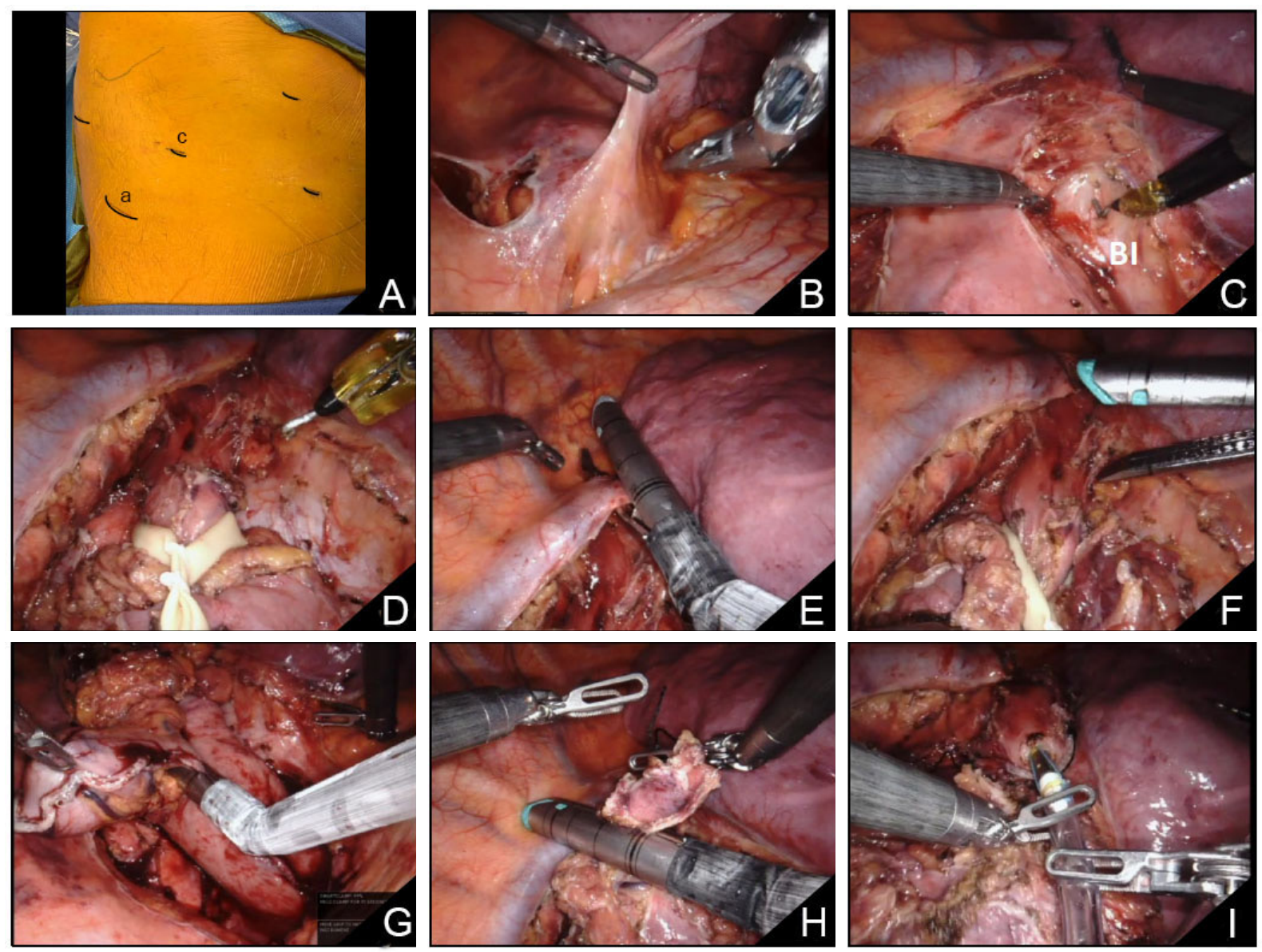

Figure 2. Thoracic portion of robotic Ivor Lewis esophagectomy. Robotic ports are placed in the right chest as shown [A: (c) camera port; (a) assistant port]; dissection in the chest typically begins with division of the inferior pulmonary ligament (B) followed by circumferential dissection of the esophagus (C) to allow placement of a Penrose drain around it (D); if performing the operation for an esophageal malignancy, nodal tissue is swept up with the specimen; the airway will be visualized during the dissection (BI). The azygos vein is divided flush with the cava (E) and the esophagus is transected superior to the azygos (F); the gastric conduit is delivered into the chest (G); and the proximal esophageal margin is checked in malignant cases $(\mathrm{H})$; a transoral anvil is then delivered through the esophageal staple line (I) and an end-to-end anastomotic stapler used to complete the anastomosis, performed extra-corporeally here (images not captured). BI: bronchus intermedius

A nasogastric tube is carefully advanced beyond the anastomosis and the gastrotomy site is resected using an endoscopic gastrointestinal anastomosis (GIA) stapler. After creation of the anastomosis, a soft tissue drain is placed adjacent to the conduit, and a chest tube is placed in the pleural cavity, prior to re-inflating the right lung under direct visualization and closure.

Of note, the esophagogastric anastomosis can be performed in any of several different manners, including a linear side-to-side (functional end-to-end) stapled or a completely sewn 2-layer technique. No particular method has proven superior in terms of anastomotic leakage, though stapled anastomoses appear to lead to fewer strictures than ones that are completely sewn ${ }^{[19,20]}$.

\section{THREE-FIELD RAMIE (MCKEOWN RAMIE)}

\section{Patient positioning and port placement}

McKeown RAMIE is started with esophageal dissection in the chest. A double lumen endotracheal tube is placed and the patient is rotated to the left lateral decubitus position. The lung is isolated and four robotic ports are used for esophageal mobilization with or without an assistant port, similar to the thoracic phase 

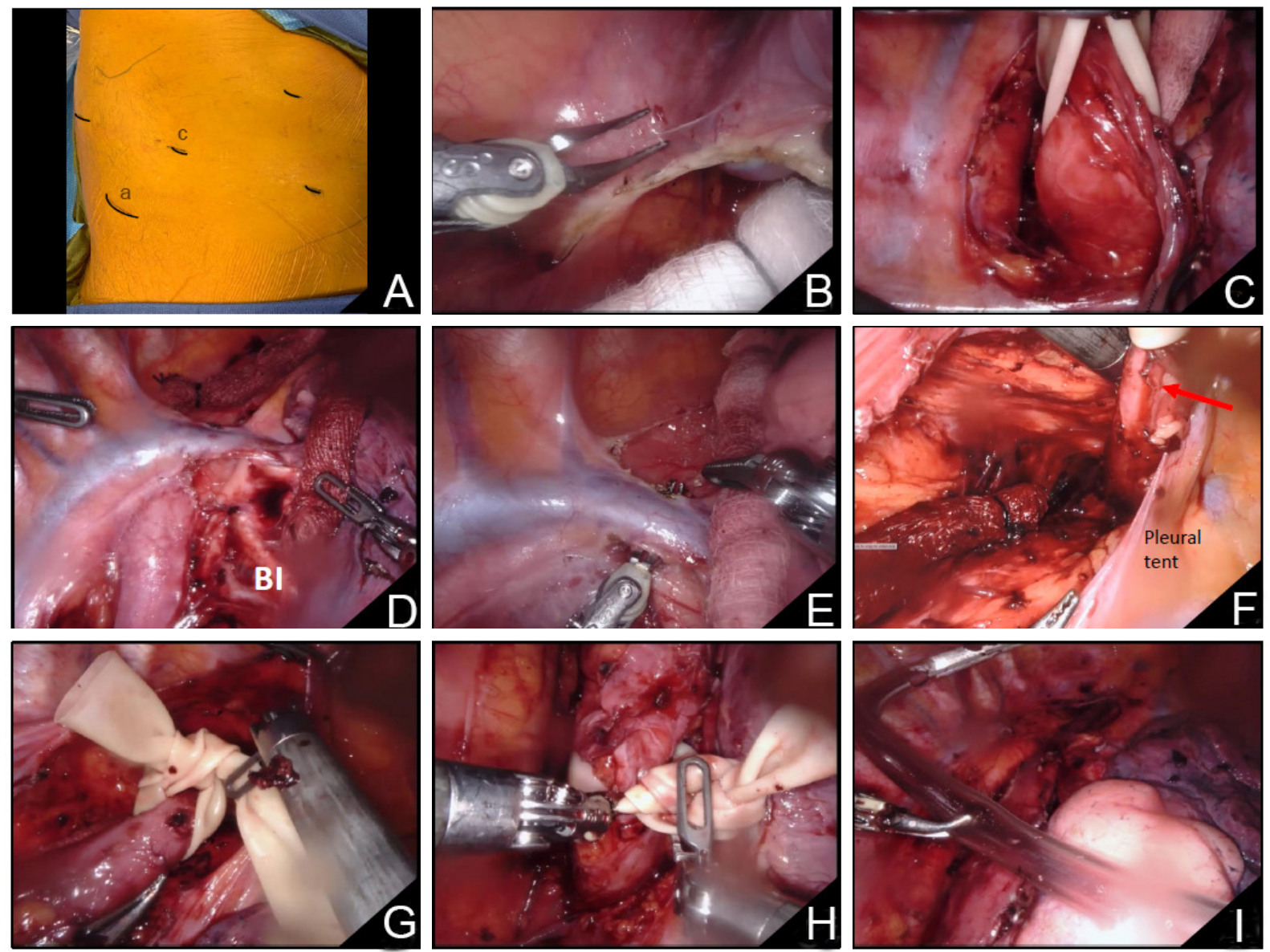

Figure 3. Thoracic portion of robotic McKeown esophagectomy. Once the ports are placed [A: (c) camera port; (a) assistant port] and the robot is docked, dissection begins at the inferior pulmonary ligament (B); as the dissection is carried en bloc superiorly, a Penrose drain is placed around the esophagus (C) to aid in retraction. The airway is visualized during skeletonization for cases of malignancy (D: $\mathrm{BI}$ ); the azygos vein (E) is divided, and dissection is carried up to the apex with creation of a pleural tent ( $F$, red arrow pointing towards the mobilized esophagus). One Penrose drain is tucked at the apex (G) while another is tucked at the diaphragm (H) prior to closing the chest (I). BI: bronchus intermedius

of an Ivor Lewis esophagectomy [Figure 3A]. Three robotic ports are placed in the eighth intercostal space - one anterior to the anterior axillary line $(12 \mathrm{~mm})$, another at the posterior axillary line $(8 \mathrm{~mm})$, and another $(8 \mathrm{~mm})$ posteriorly approximately $2 \mathrm{~cm}$ from the spine. An $8 \mathrm{~mm}$ robotic port is placed in the third or fourth intercostal space anteriorly to facilitate upper esophageal mobilization. An assistant $12 \mathrm{~mm}$ valveless insufflation port is placed low in the pleural cavity at approximately the tenth intercostal space and the chest is insufflated to a pressure of $8-10 \mathrm{mmHg}$.

\section{Thoracic portion of the procedure}

Dissection starts at the inferior pulmonary ligament and proceeds posteriorly along the esophagus [Figure 3B]. The esophagus is dissected circumferentially along with para-esophageal lymphoid tissue. A Penrose drain is placed circumferentially around the esophagus to aid superior and inferior mobilization [Figure 3C]. The airway is skeletonized in the process [Figure 3D]. Care must be taken not to injure the thoracic duct; if there is doubt, the duct should be clipped or ligated. The azygos vein is divided near the SVC using a curved tip vascular load placed through the anterior port [Figure 3E]. The previously placed Penrose drain is positioned in the apex of the chest for retrieval from the neck [Figure $3 \mathrm{~F}$ and $\mathrm{G}$ ]. A second Penrose drain can be placed at the level of the diaphragm for later retrieval from the abdomen [Figure $3 \mathrm{H}]$. After ensuring adequate hemostasis, a chest tube is placed though the anterior incision and advanced to the apex [Figure 3I]. The lung is then re-inflated under direct visualization before closing the incisions. 


\section{Abdominal dissection}

The patient is positioned supine and the double lumen endotracheal tube is switched to a single lumen tube. The patient's neck is extended and turned slightly to the right, exposing the left lower anterior neck. The neck, anterior chest, and abdomen are prepped and draped in routine sterile fashion. The abdominal ports are placed as for an Ivor Lewis RAMIE [Figure 4A]. Four robotic ports (one $12 \mathrm{~mm}$ and three $8 \mathrm{~mm}$ ) are typically employed; a $12 \mathrm{~mm}$ right upper quadrant port is needed to create a gastric conduit with the use of a robotic stapler.

The abdomen is entered with either a Hassan or Optiview technique with a 0-degree, $5 \mathrm{~mm}$ camera in the left upper quadrant. This port is later converted to an $8 \mathrm{~mm}$ robotic port. Once in the peritoneum, the abdomen is insufflated with carbon dioxide to a pressure of $15 \mathrm{mmHg}$. Three additional robotic ports (one $8 \mathrm{~mm}$ midline, one $8 \mathrm{~mm}$ left lateral quadrant, and one $12 \mathrm{~mm}$ right upper quadrant) are placed under direct visualization. These ports are placed equidistant from the xiphoid process. A $5 \mathrm{~mm}$ liver retractor port can be employed either laterally in the right upper quadrant or near the subxiphoid process. Finally, an assistant $12 \mathrm{~mm}$ valveless port is placed low in the pelvis typically on the patient's right side, in order to facilitate placement of a feeding jejunostomy in the left lower quadrant.

The dissection is started with creation of the gastric conduit. The gastrocolic ligament is divided along the greater curvature of the stomach with a robotic vessel sealer while preserving the right gastroepiploic arterial arcade [Figure 4B]. Once the right gastroepiploic trunk tapers proximally along the greater curvature, the dissection plane is moved closer to the stomach and the short gastric vessels are divided [Figure $4 \mathrm{C}$ ]. Next, posterior dissection of the stomach is completed [Figure $4 \mathrm{D}$ ], and the peritoneum over the left and right crus is stripped and swept away with the surgical specimen. Dissection is continued into the mediastinum until the inferior Penrose drain, placed during the thoracic phase of the procedure, is encountered and delivered into the surgical field.

The left gastric artery is identified and divided using a robotic vascular stapler. The pars flaccida is divided, and a $4-5 \mathrm{~cm}$ gastric conduit is created [Figure $4 \mathrm{E}$ ] with serial firings of the robotic $45 \mathrm{~mm}$ blue or green load staplers [Figure 4F]. Additional conduit length can be achieved by gently stretching the stomach longitudinally as the robotic stapler is fired. Using near-infrared imaging, IV ICG can be administered to allow identification of the transition point of perfusion along the conduit. A stitch can be placed to mark this point, guiding creation of the esophagogastric anastomosis at a region of adequate gastric perfusion [Figure $4 \mathrm{G}$ ]. Lastly, the conduit is marked to ensure delivery of the conduit to the neck without torsion as it is pulled up through the mediastinum [Figure $4 \mathrm{H}$ ].

Our practice is to perform a pyloric drainage procedure. A Heineke-Mikulicz pyloroplasty is completed as described previously and buttressed with a tongue of the omentum [Figure 4I]. A Kocher maneuver may be performed if the pylorus does not reach the diaphragmatic hiatus. A 12-14 Fr feeding jejunostomy is placed in the proximal jejunum and brought out to the skin in the left lower quadrant.

\section{Left cervical dissection and anastomotic development}

A five $\mathrm{cm}$ oblique incision is made anterior to the left sternocleidomastoid (SCM) muscle extending cephalad from the sternal notch. The platysma is divided and the left SCM is retracted laterally. Using electrocautery, the left omohyoid muscle is transected and the strap muscles divided as needed to provide exposure of the cervical esophagus. The left inferior thyroid artery may need to be clamped, divided, and ligated as it frequently impairs this exposure. Division and ligation of the vessel should be performed as far laterally as possible to avoid injury to the left recurrent laryngeal nerve (RLN). Injury to this nerve is also prevented by avoiding electrocautery and placement of either metal pickups or retractors in the region of the left tracheo-esophageal (TE) groove. The assistant's index finger, rather than a metal retractor, is 

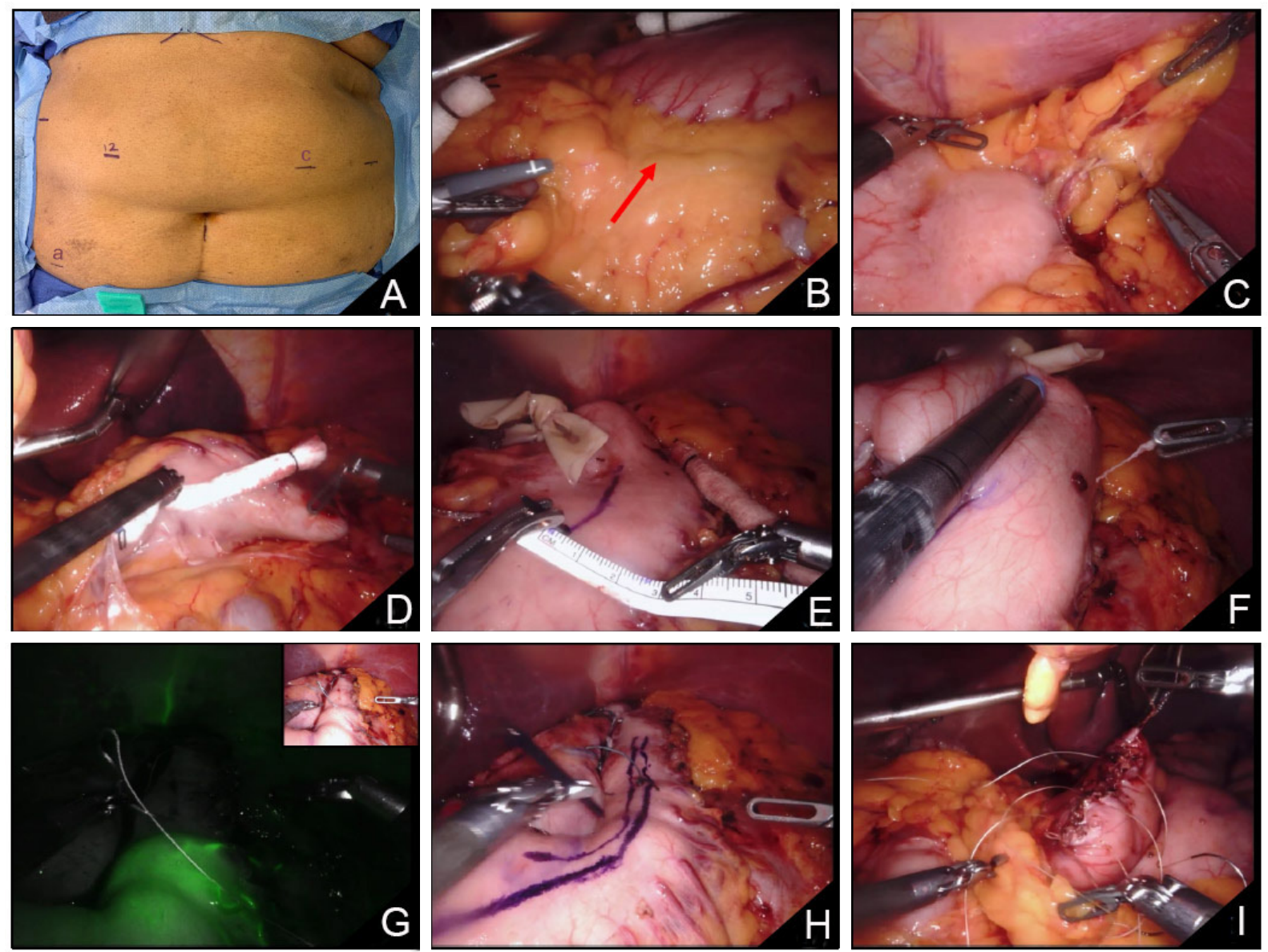

Figure 4. Abdominal portion of robotic McKeown esophagectomy. Four robotic ports are placed, along with a liver retractor in the right upper quadrant and assistant port in the right lower quadrant [A: (c) camera port; (a) assistant port]; next, the greater omentum is divided after identifying the right gastroepiploic arcade (B, red arrow) and the dissection is carried up to the short gastric vessels (C); the stomach is rotated to the right and posterior attachments are divided (D); after ensuring circumferential dissection of the conduit, the mediastinal Penrose drain is delivered into the field (E); a 4-5 cm gastric conduit is created ( $E$ and $F$ ) and indocyanine green testing of the conduit is conducted prior to transecting the stomach $(\mathrm{G})$; a transition stitch can be placed where there is a clear demarcation in perfusion ( $\mathrm{G}$ insert); once the stomach is transected, two parallel lines are marked on the conduit to ensure that the conduit is pulled into the neck without torsion $(\mathrm{H})$; a pyloric drainage procedure may be performed (I). The anastomosis is performed in the neck below the transition stitch (images not captured)

utilized to retract the trachea and improve exposure of the cervical esophagus. A Henley retractor with interchangeable blades of varying lengths is the optimal tool for facilitating exposure in the neck. A long blade attached to the Henley is used to retract the carotid sheath, and a shorter blade on the opposite arm is used to retract the strap muscles. As previously mentioned, a retractor blade should not be placed in the TE groove to prevent RLN injury. The esophagus is then mobilized in a circumferential fashion using a combination of blunt and sharp dissection. The dissection plane is kept on the surface of the esophagus, taking care to avoid the left RLN situated more anteriorly toward the trachea. Once the esophagus has been mobilized in its entirety, the apical Penrose drain that was placed during the thoracic phase of the procedure is manually palpated and delivered into the incision.

The specimen is then pulled up gradually into the neck. Direct robotic visualization from the abdomen should be performed while the conduit is carefully delivered through the mediastinum. Care must be taken to ensure that the conduit does not torse as it gets pulled up into the mediastinum. Once the conduit is completely delivered and the transition stitch is identified, either a linear side-to-side (functional end-toend) or a completely handsewn anastomosis is performed. We prefer the former approach utilizing the GIA 
stapler. The common opening may be closed with either a TA stapler or using a hand-sewn technique. A nasogastric tube is passed under direct visualization before completing the anterior wall of the anastomosis. A drain is left in the cervical bed to monitor for leaks. After placement of two 2-0 sutures securing the conduit to the diaphragm, pneumoperitoneum is reduced, and the abdominal and neck incisions closed in layers.

\section{TRANSHIATAL RAMIE}

Typically, transhiatal RAMIE is chosen for patients who have mitigating pulmonary or cardiac comorbidities and may not tolerate single-lung ventilation. Transhiatal RAMIEs can be technically challenging given the mediastinal dissection with the robot.

\section{Patient positioning and port placement}

The patient is positioned supine, with a single lumen tube, with both arms tucked. The neck is turned to the patient's right, and the left neck, chest, abdomen, and pelvis are all prepped and draped in one field. The robot is docked in the abdomen. Typically, four robotic ports (one $12 \mathrm{~mm}$ and three $8 \mathrm{~mm}$ ) are employed. The $12 \mathrm{~mm}$ port is positioned in the right upper quadrant to allow creation of the gastric conduit with the use of a robotic stapler. The abdomen is entered either using a Hassan or an Optiview technique with a o-degree, $5 \mathrm{~mm}$ camera in the left upper quadrant. This port is later converted to an $8 \mathrm{~mm}$ robotic port. Once in the peritoneum, the abdomen is insufflated with carbon dioxide to a pressure of $15 \mathrm{mmHg}$. Three remaining robotic ports (one $8 \mathrm{~mm}$ midline, one $8 \mathrm{~mm}$ left lateral quadrant, and one $12 \mathrm{~mm}$ right upper quadrant) are employed under direction visualization and are positioned equidistant from the xiphoid process. A $5 \mathrm{~mm}$ liver retractor port can either be placed laterally in the right upper quadrant or near the subxiphoid process. Lastly, an assistant port is positioned low in the pelvis, typically on the patient's right side, to facilitate placement of a feeding jejunostomy in the left lower quadrant.

\section{Abdominal portion of the procedure}

The abdominal portion begins with creation of the gastric conduit. Gastric mobilization starts with dividing the greater omentum and preserving the right gastroepiploic arcade. Short gastric vessels are divided, and the stomach is rotated anteriorly to allow take-down of retrogastric adhesions. Dissection is then carried over to the lesser omentum. The pars flaccida is opened and the incision extended toward the right crus. The left gastric and celiac axis nodes are swept up towards the specimen. Hiatal dissection is completed circumferentially while stripping the peritoneum off the crura.

Under direct visualization, the camera and instruments are advanced into the mediastinum for extensive mediastinal dissection. Dissection is continued as high as possible in order to facilitate mobilization of the native esophagus from the neck, which is blind otherwise.

A 4-5 cm gastric conduit is created utilizing sequential stapler fires along the lesser curve. Starting at the incisura angularis, a vascular load is first employed, followed by serial firing of blue- or green- robotic staplers while applying gentle longitudinal tension on the conduit. Once the esophagus is completely transected, the conduit is attached to the specimen for later retrieval in the neck. A Heineke-Mikulicz pyloroplasty is completed by placing stay sutures on the superior and inferior aspects of the pylorus. The pylorus is opened longitudinally and closed transversely, ensuring mucosal apposition during closure. The suture line can be covered with a tongue of omentum. A generous Kocher maneuver can be performed if the pylorus does not reach the hiatus easily to allow for tension-free delivery of the anastomotic site into the neck. A 12-14 Fr jejunostomy feeding tube is placed in the left lower quadrant.

\section{Cervical dissection and anastomotic development}

With the abdominal ports in place, attention is diverted to the left neck. A $5 \mathrm{~cm}$ oblique incision is made anterior to the left SCM. Dissection is carried down through the platysma using electrocautery. The 
omohyoid muscle is divided, the inferior thyroid artery ligated and divided, and the strap muscles dissected to allow access to the esophagus. No cautery or metal retractors are used in the region of the TE groove to avoid inadvertent injury to the RLN. Blunt and sharp dissection is performed circumferentially around the cervical esophagus. If dense adhesions are encountered at the thoracic inlet, a mediastinoscope can be employed via the cervical incision to facilitate adhesiolysis under direct visualization. Such efforts allow precise dissection and prevent inadvertent injury to the azygos. Next, the specimen and conduit are pulled into the neck in proper orientation, and the stomach is divided at or below the transition stitch. The proximal and distal margins are checked for metaplasia, dysplasia, or malignancy prior to completing the anastomosis.

The anastomosis is performed either in a completely hand-sewn fashion or by utilizing a hybrid technique, whereby the posterior aspect is started with a GIA stapler and the common opening is closed anteriorly in a hand-sewn fashion. A nasogastric tube is passed under direct visualization prior to closing the anterior wall. A drain is placed in the neck to monitor for leaks. The conduit is then tacked to the crura with two 2-0 Ethibond sutures. After ensuring hemostasis, pneumoperitoneum is released, and all incisions are closed in layers.

\section{POSTOPERATIVE COURSE}

After surgery, the patient is admitted to an intensive or intermediate care unit for one to two days and stepped down to a regular surgical unit once clinically stable. Enteral feeds are started via the feeding jejunostomy tube on postoperative day 1 and advanced to goal over the course of the next few days. The nasogastric tube is discontinued after return of bowel function and when output is at an acceptably low level. The chest tube is typically removed on postoperative day 4 or 5 , depending on the volume and character of drainage. The patient is discharged once they have reached their benchmarks and are tolerating goal tube feeds. The patient is kept NPO until a swallow study is performed as an outpatient on postoperative day 14 . This protocol promotes early discharge while allowing small, clinically insignificant anastomotic leaks to seal.

\section{LYMPH NODE DISSECTION IN RAMIE}

The extent of lymph node dissection has been an important topic in the thoracic surgical literature. Unlike resections for colorectal cancer, no definite cut-off has been established to define adequate lymph node harvest for esophageal or esophagogastric junction carcinoma; different reports have determined varying thresholds. Of importance is the fact that the aggregate lymph node count does not take into consideration the location of the nodal basins harvested, such as whether they are in the abdomen, chest, or neck. A better measure of the adequacy of lymphadenectomy, therefore, is the rate of locoregional recurrence following esophagectomy by the various approaches.

Recent large cohort studies have found an average harvest of 25-29 regional nodes during RAMIE ${ }^{[15,21,22]}$. Rates of locoregional recurrence, however, are not well defined, as the studies do not differentiate local and distant recurrences when determining disease-free survival. When compared to open or traditional MIE, locoregional recurrence rates following RAMIE have been reported to be comparable or lower ${ }^{[5,23]}$. Robotic surgical platforms may offer advantages in dissection along the RLN in the apex of the chest, performed with the patient in either the prone or lateral decubitus position ${ }^{[24,25]}$. A number of publications have also confirmed the lower incidence of RLN neuropraxia and vocal cord paralysis with RAMIE when compared to traditional $\mathrm{MIE}^{[26,27]}$.

\section{DISCUSSION}

With increasing exposure to robotic surgical techniques and with continual improvements in robotic design and technology, including the introduction of robotic staplers and energy devices, the number of 
robotic cancer operations is on the rise ${ }^{[28,29]}$. A major advantage of current robotic systems, compared to the performance of an open esophagectomy, is the seven degrees-of-freedom in the wristed instruments, allowing the surgeon to operate ergonomically with angulation comparable to the human wrist. This dexterity allows the surgeon to complete esophageal and nodal dissections similar to an open procedure, facilitates intracorporeal suturing and knot tying, and enhances the surgeon's ability to operate in difficultto-reach places such as the apex of the chest, subcarinal space, and splenic flexure. The additional advantages of current robotic platforms include three-dimensional visualization, 10-fold magnification, and a 6-Hertz motion filter designed to eliminate tremor. In addition, robotic surgical platforms offer longer instruments compared to other minimally invasive systems with a fixed fulcrum supported by robotic arms, potentially leading to reduced stress on the chest and abdominal wall. An operating console for a second surgeon is used in some robotic operating rooms, allowing surgeons to perform surgery in tandem with, and facilitating training in a dynamic and supportive manner.

Given these advantages, the increasing utilization of surgical robotics in the performance of esophagectomy should come as no surprise. While the initial experiences with RAMIE were associated with higher complication rates, subsequent reports have shown that RAMIE can be performed with superior perioperative outcomes, and equivalent oncologic survival when compared to open and traditional minimally invasive approaches ${ }^{[14,15]}$. Such experiences suggest that the potential disadvantages of current surgical robots, including the lack of haptic feedback and the positioning of the surgeon at a remote console in a non-sterile environment, can be mitigated by surgeon experience and the presence of trained assistants in the sterile field. The incidence of associated complications, such as major uncontrolled hemorrhage, appears to be at an acceptably low level.

\section{Ongoing studies about RAMIE}

Numerous retrospective studies have supported the role of MIE and RAMIE when compared to open esophagectomy. Only a limited number of prospectively designed, randomized controlled trials (RCTs), however, have been reported to date. An RCT of 115 patients from 5 European centers (the TIME trial) evaluated the outcomes of 59 patients randomly assigned to MIE compared to 56 patients randomized to open esophagectomy. Initial results were published in 2012, and long-term results in $2017^{[1,4]}$. While the baseline demographics and clinical characteristics were similar in both groups, the overall 3-year survival was higher in the MIE group (50.5\% vs. 40.4\%), although the difference was not statistically significant $(P=$ 0.207). Disease-free 3-year survival rates were also similar between the two groups [ $40.2 \%$ for MIE, $35.9 \%$ for open; $\mathrm{HR}=0.691$ (95\%CI: 0.389-1.239)]. Of note, pulmonary complications were significantly lower in the MIE group ( $12 \% v s .34 \%, P=0.005)$, as was blood loss $(200 \mathrm{~mL} v s .475 \mathrm{~mL}, P<0.001)$ and hospital stay (11 days vs. 14 days, $P=0.044$ ) despite conversions from MIE to open in eight cases. The anastomotic leak rate, re-operative rates, and 30-day mortality rates were similar between groups.

The ROBOT trial, published in 2019, was an RCT from a single institution in the Netherlands ${ }^{[5]}$. Patients with esophageal cancer were randomized to RAMIE $(n=54)$ or open esophagectomy $(n=55)$. Findings were similar to those from TIME, with less overall surgery-related complications following RAMIE (59\% vs. $80 \%$; $P=0.02)$, fewer pulmonary complications ( $32 \%$ vs. $58 \% ; P=0.005)$, and a lower incidence of atrial fibrillation $(22 \% v s .46 \%, P=0.01)$. On the contrary, no differences were noted in anastomotic leak rates or mortality rates between the two groups. Median ICU stay, hospital stay, Ro resection rates, and lymph node retrieval numbers were not significantly different between the two groups. Functional recovery, patient reported pain scores, and short-term quality of life assessments all favored the RAMIE approach. Overall, the study found improved short-term outcomes following robotic esophagectomy compared to the open approach.

While several retrospective studies, as well as these RCTs, have compared both MIE and RAMIE to open esophagectomy, no studies have compared RAMIE to MIE. A recently opened trial (RAMIE trial) is 
designed to compare RAMIE to MIE in a randomized controlled setting ${ }^{[30]}$. The study includes four centers from China and will focus on patients with esophageal squamous cell carcinoma (SCC). The primary endpoint of the study is 5-year overall survival, with secondary endpoints of 3-year overall and disease-free survival, 5-year disease-free survival, short-term outcomes, and quality of life. The hypothesis of the trial is that RAMIE will result in equivalent oncologic outcomes and long-term quality of life, along with shorter operative times, lower perioperative complications, and shorter hospital stays, when compared to MIE.

The REVATE trial, a two-center, open-label RCT of esophageal SCC, will compare lymph node dissection along the RLN during RAMIE $v s$. MIE ${ }^{[25]}$. The two institutions participating in this RCT are from China and Taiwan, where SCC is more prominent than esophageal adenocarcinoma, favoring upper or middle esophageal tumors where dissection of lymph nodes around the RLNs is critical. The study, while meaningful for that patient population, will be difficult to apply to Western cohorts comprised primarily of patients with adenocarcinoma of the distal esophagus or esophagogastric junction.

\section{CONCLUSION}

Robotic approaches to esophagectomy are being utilized with increased frequency and improve upon established techniques of MIE by offering superior dexterity, maneuverability, ergonomics, and visualization. Randomized and non-randomized studies have demonstrated equivalent oncologic outcomes between robotic and open esophagectomies, with superior results for the robotic approach in terms of hospital stay, postoperative morbidity, and overall quality of life. As surgeons become trained in robotic techniques and implement them further into their treatment armamentarium, the use of RAMIE for appropriately selected cases of esophageal malignancy or other end-stage esophageal disease will undoubtedly, continue to increase. With the introduction of novel and competing robotic technologies, the hope is that their cost will decrease, allowing further penetration into the marketplace.

\section{DECLARATIONS}

\section{Authors' contributions}

Made substantial contributions to the conception and design of the manuscript, and performed data analysis and interpretation: Khaitan PG

Contributed to manuscript writing and editing: Lazar JF, Henderson HR, Watson TJ

Provided technical and material support: Margolis M

\section{Availability of data and materials}

Not applicable.

\section{Financial support and sponsorship}

None.

\section{Conflicts of interest}

All authors declared that there are no conflicts of interest.

\section{Ethical approval and consent to participate}

Not applicable.

\section{Consent for publication}

Not applicable.

\section{Copyright}

(c) The Author(s) 2020. 


\section{REFERENCES}

1. Biere SS, van Berge Henegouwen MI, Maas KW, Bonavina L, Rosman C, et al. Minimally invasive versus open oesophagectomy for patients with oesophageal cancer: a multicentre, open-label, randomised controlled trial. Lancet 2012;379:1887-92.

2. Puntambekar S, Kenawadekar R, Kumar S, Joshi S, Agarwal G, et al. Robotic transthoracic esophagectomy. BMC Surg 2015;15:47.

3. Safranek PM, Cubitt J, Booth MI, Dehn TC. Review of open and minimal access approaches to oesophagectomy for cancer. Br J Surg 2010;97:1845-53.

4. Straatman J, van der Wielen N, Cuesta MA, Daams F, Roig Garcia J, et al. Minimally invasive versus open esophageal resection: threeyear follow-up of the previously reported randomized controlled trial: the TIME trial. Ann Surg 2017;266:232-6.

5. van der Sluis PC, van der Horst S, May AM, Schippers C, Brosens LAA, et al. Robot-assisted minimally invasive thoracolaparoscopic esophagectomy versus open transthoracic esophagectomy for resectable esophageal cancer: a randomized controlled trial. Ann Surg 2019;269:621-30.

6. Collard JM, Lengele B, Otte JB, Kestens PJ. En bloc and standard esophagectomies by thoracoscopy. Ann Thorac Surg 1993;56:675-9.

7. Melvin WS, Needleman BJ, Krause KR, Schneider C, Wolf RK, et al. Computer-enhanced robotic telesurgery. Initial experience in foregut surgery. Surg Endosc 2002;16:1790-2.

8. Horgan S, Berger RA, Elli EF, Espat NJ. Robotic-assisted minimally invasive transhiatal esophagectomy. Am Surg 2003;69:624-6.

9. Kernstine KH, DeArmond DT, Karimi M, Van Natta TL, Campos JH, et al. The robotic, 2-stage, 3-field esophagolymphadenectomy. J Thorac Cardiovasc Surg 2004;127:1847-9.

10. van Hillegersberg R, Boone J, Draaisma WA, Broeders IA, Giezeman MJ, et al. First experience with robot-assisted thoracoscopic esophagolymphadenectomy for esophageal cancer. Surg Endosc 2006;20:1435-9.

11. Kernstine KH, DeArmond DT, Shamoun DM, Campos JH. The first series of completely robotic esophagectomies with three-field lymphadenectomy: initial experience. Surg Endosc 2007;21:2285-92.

12. Dunn DH, Johnson EM, Morphew JA, Dilworth HP, Krueger JL, et al. Robot-assisted transhiatal esophagectomy: a 3-year single-center experience. Dis Esophagus 2013;26:159-66.

13. Cerfolio RJ, Wei B, Hawn MT, Minnich DJ. Robotic esophagectomy for cancer: early results and lessons learned. Semin Thorac Cardiovasc Surg 2016;28:160-9.

14. He H, Wu Q, Wang Z, Zhang Y, Chen N, et al. Short-term outcomes of robot-assisted minimally invasive esophagectomy for esophageal cancer: a propensity score matched analysis. J Cardiothorac Surg 2018;13:52.

15. Tagkalos E, Goense L, Hoppe-Lotichius M, Ruurda JP, Babic B, et al. Robot-assisted minimally invasive esophagectomy (RAMIE) compared to conventional minimally invasive esophagectomy (MIE) for esophageal cancer: a propensity-matched analysis. Dis Esophagus 2020;33:doz060.

16. van der Sluis PC, Ruurda JP, van der Horst S, Goense L, van Hillegersberg R. Learning curve for robot-assisted minimally invasive thoracoscopic esophagectomy: results from 312 cases. Ann Thorac Surg 2018;106:264-71.

17. Ladak F, Dang JT, Switzer N, Mocanu V, Tian C, et al. Indocyanine green for the prevention of anastomotic leaks following esophagectomy: a meta-analysis. Surg Endosc 2019;33:384-94.

18. Okusanya O, Lu M, Luketich JD, Sarkaria IS. Intraoperative near infrared fluorescence imaging for the assessment of the gastric conduit. J Thorac Dis 2019;11:S750-4.

19. Plat VD, Stam WT, Schoonmade LJ, Heineman DJ, van der Peet DL, et al. Implementation of robot-assisted Ivor Lewis procedure: Robotic hand-sewn, linear or circular technique? Am J Surg 2020;220:62-8.

20. Zhang H, Wang Z, Zheng Y, Geng Y, Wang F, et al. Robotic side-to-side and end-to-side stapled esophagogastric anastomosis of ivor lewis esophagectomy for cancer. World J Surg 2019;43:3074-82.

21. Sarkaria IS, Rizk NP, Goldman DA, Sima C, Tan KS, et al. Early quality of life outcomes after robotic-assisted minimally invasive and open esophagectomy. Ann Thorac Surg 2019;108:920-8.

22. van der Sluis PC, Tagkalos E, Hadzijusufovic E, Babic B, Uzun E, et al. Robot-assisted minimally invasive esophagectomy with intrathoracic anastomosis (Ivor Lewis): promising results in 100 consecutive patients (the European experience). J Gastrointest Surg 2020; doi: 10.1007/s11605-019-04510-8.

23. Na KJ, Park S, Park IK, Kim YT, Kang CH. Outcomes after total robotic esophagectomy for esophageal cancer: a propensity-matched comparison with hybrid robotic esophagectomy. J Thorac Dis 2019;11:5310-20.

24. Oshikiri T, Yasuda T, Harada H, Goto H, Oyama M, et al. A new method (the "Bascule method") for lymphadenectomy along the left recurrent laryngeal nerve during prone esophagectomy for esophageal cancer. Surg Endosc 2015;29:2442-50.

25. Chao YK, Li ZG, Wen YW, Kim DJ, Park SY, et al. Robotic-assisted esophagectomy vs video-assisted thoracoscopic esophagectomy (REVATE): study protocol for a randomized controlled trial. Trials 2019;20:346.

26. Chao YK, Wen YW, Chuang WY, Cerfolio RJ. Transition from video-assisted thoracoscopic to robotic esophagectomy: a single surgeon's experience. Dis Esophagus 2020;33:doz033.

27. Chen J, Liu Q, Zhang X, Yang H, Tan Z, et al. Comparisons of short-term outcomes between robot-assisted and thoraco-laparoscopic esophagectomy with extended two-field lymph node dissection for resectable thoracic esophageal squamous cell carcinoma. J Thorac Dis 2019;11:3874-80.

28. Konstantinidis IT, Ituarte P, Woo Y, Warner SG, Melstrom K, et al. Trends and outcomes of robotic surgery for gastrointestinal (GI) cancers in the USA: maintaining perioperative and oncologic safety. Surg Endosc 2019; doi: 10.1007/s00464-019-07284-x.

29. Sheetz KH, Claflin J, Dimick JB. Trends in the adoption of robotic surgery for common surgical procedures. JAMA Netw Open 2020;3:e1918911. 
30. Yang Y, Zhang X, Li B, Li Z, Sun Y, et al. Robot-assisted esophagectomy (RAE) versus conventional minimally invasive esophagectomy (MIE) for resectable esophageal squamous cell carcinoma: protocol for a multicenter prospective randomized controlled trial (RAMIE trial, robot-assisted minimally invasive Esophagectomy). BMC Cancer 2019;19:608. 